\title{
Humanidades Médicas: Un Enfoque Novedoso Sobre Medicina Para El Condado De Washington, Utah
}

\author{
Rachel Manuele, BA Biomedical Sciences and Literary Studies ${ }^{1}{ }^{a}$, Koy Gubler, Translator ${ }^{1}$ \\ ${ }^{1}$ Dixie State University \\ Keywords: medico las letras, la medicina \\ https://doi.org/10.36898/001c.22020
}

Curiosity: Interdisciplinary Journal of Research and Innovation

Vol. 2, 2021

\begin{abstract}
Para amplificar y enriquecer la educación de los médicos, las humanidades se han introducido en el currículo de la escuela de medicina a través de la literatura, la historia, el arte e incluso la música. La cultura médica actual requiere e incluso provoca la desvinculación emocional de los proveedores del sistema médico, pero las humanidades proporcionan una metodología para cerrar esa brecha lamentable entre el paciente y médico. El cierre de esta brecha romperá las barreras que impiden el servicio excepcional del sistema médico. La fusión de humanidades médicas en la educación y la práctica de los médicos proporciona no sólo una metodología, sino una cultura que se puede incorporar dentro al sistema médico. La adopción de esta cultura en el condado de Washington, Utah, podría ayudar a cerrar la brecha, beneficiando en gran medida a los ciudadanos y proveedores del sistema médico por igual. Este proyecto de investigación, que incluye entrevistas con miembros de la comunidad de St. George y la Universidad de Utah, sugiere los beneficios que un enfoque interdisciplinario puede tener para el condado de Washington en sus hospitales y clínicas, también en el currículo de estudios universitarios y en la escuela de medicina.
\end{abstract}

En el sistema de salud actual, la brecha que se siente entre el paciente y el médico no tiene nada que ver con las preguntas perpetuas que rodean el costo y el seguro. En cambio, está en las interacciones entre las partes, articuladas e implícitas. Tampoco es solo un problema de comunicación, aunque ese es un indicador importante de la existencia de la brecha. El corazón de ella, sin embargo, se sumerge mucho más profundo. La brecha que enfrentan ambos lados de la atención médica está en el concepto subyacente detrás de la propia medicina, algo que tanto el paciente como el médico están buscando: la curación.

Por supuesto, curar heridas físicas es el oficio del médico, pero el concepto más abstracto de lo que significa sanar y ser curado implica mucho más que tomar recetas recomendadas o envolver un esguince muscular. Para tratar al paciente, para curar al paciente, la atención médica tiene que centrarse la manera de tratar un paciente en su totalidad. El sistema de salud de hoy está tan enrevesado que hay una necesidad nacional, si no universal, de innovación. Desafortunadamente, eso implica tantos detalles legales y políticos intrincados que aquellos que quieren hacer un cambio están perdidos en cuanto a cómo

\footnotetext{
a Rachel Manuele graduated May 2020 from Dixie State University with B.A. degrees in Biomedical Sciences and Literary Studies. She is currently applying to graduate schools to earn her Masters of Science to become a physician assistant. Rachel has been involved with DSU's Booth Honors Program, the American Medical Women's Association, Dixie Pre-Medical Alliance, The Southern Quill, and Sigma Tau Delta.
} 
hacerlo, y nadie puede ponerse de acuerdo sobre qué método es el más beneficioso. Por lo tanto, mientras esa pregunta sigue en pie, las escuelas de medicina en todo el mundo están utilizando diferentes métodos para capacitar a los futuros médicos no sólo cómo practicantes la medicina, o cómo ser buenos médicos, sino cómo curar al paciente en su totalidad mediante la incorporación de un enfoque holístico del tratamiento médico. La esperanza es que estos médicos lleven estas enseñanzas a sus prácticas y revolucionen la cultura actual de la medicina. Para ello, las escuelas de medicina están implementando un cruce de disciplina único en sus currículos de estudio mediante la incorporación de las humanidades en la educación médica de sus estudiantes.

Hasta hace poco, la cultura médica requería e incluso fomentaba el desprendimiento emocional de sus proveedores de atención médica. A un médico se le enseñó este distanciamiento explícitamente como "preocupación distanciada" en la escuela de medicina (Weiner, 2019, párr. 15). Como resultado de la "preocupación distanciada", tanto el paciente como el médico sufren. Como este artículo demostrará, los pacientes se están frustrando cada vez más con la atención médica y gastan demasiado tiempo, esfuerzo y dinero para encontrar un proveedor que se preocupa. Mientras tanto, los médicos se están desanimando, quemando y deprimiendo por la falta de significado que sienten en sus trabajos.

La incorporación de las humanidades proporciona una metodología para cerrar esa brecha demasiado ancha entre paciente y médico. Las humanidades médicas pueden ayudar a cerrar esta brecha para romper las barreras que impiden la prestación de atención médica excepcional. El enfoque interdisciplinario de la combinación de medicinas y humanidades también proporciona una cultura incorporable al sistema de salud. Utilizando metodologías de humanísticas como la compasión, perspectiva y esperanza, los proveedores médicos pueden hacer las conexiones que sean necesarias para centrarse en la importancia de la curación de un individuo. La adopción de esta cultura en la educación y la práctica de los médicos en el condado de Washington, Utah, podría cerrar las brechas donde existen para proporcionar la mejor atención médica posible, beneficiando en gran medida a los ciudadanos y proveedores de atención médica por igual.

\section{La cultura histórica y actual de la medicina}

"El primer grito de dolor a través de la selva primitiva fue la primera llamada por un médico"

(Robinson, 1931, p. 1)

El fallecido médico y profesor de historia médica Victor Robinson, M.D. (1931) describió la medicina como "[un] arte natural, concebido en simpatía y nacido por necesidad” (p. 1). Su descripción de la historia médica temprana en La bistoria de la medicina pinta una imagen de una comprensión primitiva de la curación. El hombre primigenio no entendía la fisiopatología de la 
enfermedad. En lugar de pruebas que confirman que un paciente estaba libre de infección, la superstición gobernó las prácticas médicas (pp. 1-10). Debido a que la curación se consideraba divina, ningún humano podría acreditarse la recuperación de ningún paciente. Si un paciente mejoraba, debía haber sido por los dioses. Si el paciente empeoraba, la enfermedad era un castigo divino debido a un mal carácter o algún tipo de maldición (Sontag, 1977, p. 43). La simplicidad de la salud y la completa ignorancia del conocimiento médico de estos curanderos tempranos debe asombrar a los médicos de hoy en día. Sin embargo, una filosofía notable de la medicina histórica fue la conexión entre la curación y la espiritualidad.

Desde el hombre de la medicina de las tribus antiguas y los dioses de Grecia, la práctica de la medicina siempre ha abarcado más que la lógica, experimentos y deducciones en las que la ciencia se pare con orgullo. De hecho, la división entre la medicina y la magia se desdibuja considerablemente a lo largo de la historia (Robinson, 1931, p. 12). Con el avance de la tecnología y la ciencia, los rituales y encantos fueron reemplazados por hechos y teorías de biología, fisiología, patología. La ciencia ha corregido un gran número de teorías incorrectas hechas en el pasado; sin embargo, la fe y la esperanza siempre han sido parte integral de la sanación de los afligidos (p. 35).

Esculapio - indiscutiblemente el dios griego de la medicina o una persona real que simplemente albergaba seguidores de culto - era sin embargo conocido y respetado en la historia médica griega y romana; a los romanos, se hizo conocido como Esculapios (“Aesculapius," 1922, pp. 882-883; Robinson, 1931, p. 33). Incluso hoy en día, el símbolo de la curación y la medicina, la serpiente - cuyo veneno se consideraba sagrado y se utilizaba con frecuencia en las antiguas recetas griegas - entrelazada alrededor de un bastón está conectada a Esculapio y todavía se ve en hospitales y otros servicios médicos. Un bastón con dos serpientes aladas es el caduceo de Apolo, el padre de Esculapio, que era el dios de la poesía y también de la medicina, aunque la poesía era considerada la más prestigiosa de la época (Clary, 2010, p. 796; "The Caduceus of Mercury and the Staff of Aesculapius,” 1924, p. 794). Los templos ornamentados de Esculapio fueron erigidos cerca de "manchas favorecidas por la naturaleza", como supuestos muelles curativos, bosques y montañas, a los que pacientes y familias afectadas por enfermedades daban "metales preciosos, piedras costosas, terracota o cera" como pago por una cura (Robinson, 1931, p. 34). "Todas las glorias del arte griego estaban alli”", describe Robinson sobre estos costosos templos, "Y cuando la música oculta flotaba sobre las flores del sur... muchos enfermos levantaban la cabeza para repetir... oh, Esculapios, naces para ser la gran alegría del mundo” (p. 35). Como se muestra en esta escena, la música, el arte y la naturaleza son importantes en el proceso de curación. Ya que traen esperanza a los enfermos.

La ciencia ha descubierto más tarde que la música es eficaz para aliviar el dolor, incluso reduciendo el uso de opioides por parte de los pacientes, y que la poesía activa una respuesta única al centro de recompensa primaria del cerebro (Linggonegoro, 2018, párr. 3; Wassiliwizky et al., 2017, pp. 1229-1230, 
1237-1239). Aunque los pacientes son receptivos a la música en entornos clínicos han sido estudiadas (Huang et al., 2010) y se han encontrado eficaces en el tratamiento y la terapia, sólo la poesía proporcionó esperanza donde otras formas de terapia artística como la música no lo hicieron (Linggonegoro, 2018, párr. 3). "Al igual que los encantamientos antiguos", explica Clary (2010), "los poemas están destinados a ser potentes, memorables... para ser utilizados en la interpretación, revelación y sanación" (p. 796). Pero si bien los antiguos griegos y romanos no tenían la tecnología que tenemos ahora, parece que tenían mucha esperanza y fe en algo que creían que era mucho más poderoso que la medicina practicada por el hombre (p. 35). De hecho, no fue hasta que Hipócrates llegó a la escena que la superstición y la religión incuestionable se entrelazaron con la medicina y la curación.

Hipócrates se llama el Padre de la medicina porque fundó muchas prácticas y escuelas de pensamiento que los médicos todavía usan hoy en día - separó la medicina de los dioses, dándose cuenta de que la enfermedad no dependía de causas sobrenaturales (como era la creencia en ese momento), escribió historias de casos, enseñó la manera de acostarse, practicó la medicina a través del uso de medicamentos similares a los farmacéuticos, comprendió la importancia de la dieta y el clima en la salud de una persona; incluso reconoció sus fracasos en su trabajo y los denunció por la mejora continua de la medicina (Robinson, 1931, p. 54). No es de extrañar, entonces, que el Juramento Hipocrático fuera inspirado por él. En este juramento, la medicina no sólo se considera una ciencia, sino un arte (Linggonegoro, 2018). Sin embargo, a medida que la tecnología y la ciencia avanzaban, el significado y la implicación del "arte” de la medicina pueden haber sido alterados con el tiempo.

No obstante, gracias a los avances tecnológicos, aquellos que alguna vez habrían muerto sin un tratamiento científico adecuado ahora pueden vivir vidas más largas, saludables y felices. Sin embargo, una consecuencia de este avance en el conocimiento ha causado un cambio cultural completo de confiar en la superstición para explicar lo inexplicable al enfoque basado en la evidencia de los síntomas y las enfermedades. Este cambio cultural ha proporcionado una gran cantidad de conocimiento y tratamiento que salva vidas, pero si esto es o no a expensas de cosas como la esperanza y la fe, que son parte integral de la curación, puede que nunca los sepamos. La ciencia, sin embargo, está reconociendo cómo la poesía, la literatura y la música pueden aumentar la esperanza y, por lo tanto, la curación. A la luz de esto, tal vez haya algo en esa interconexión con las humanidades y la ciencia en la antigüedad que permitió a nuestros antepasados vivir incluso a pesar del insuficiente conocimiento médico (Linggonegoro, 2018). A medida que las humanidades están empezando a abrirse camino en el mundo médico actual, tanto el paciente como el médico están empezando a ver sus beneficios.

\section{Sanación a través de las humanidades}

"Aunque algunos de nosotros podamos sentirnos como fragmentos o piezas, usamos el lenguaje para encontrar y abrazar una vez más nuestras identidades, quiénes somos" 
The National Endowment for Humanities (NEH) ha financiado varios programas en todo el país para involucrar a las personas en las humanidades. Una de las poblaciones afectadas por esto es, tal vez sorprendentemente, los veteranos. Diálogos sobre las experiencias de la guerra es una rama del programa Standing Together (Estamos juntos) que "reúne a veteranos en torno a la literatura, el cine y los ejercicios de escritura reflexiva que exploran el viaje de casa al campo de batalla y de vuelta" (Muir, 2018). Para los veteranos de guerra, su dolor y sufrimiento pueden no atribuirse necesariamente a una herida de guerra física, como una amputación. En cambio, la curación que estos pacientes necesitan es algo más abstracto, desagradable y difícil de entender.

El catedrático emérito de Mellon y presidente del NEH, William Adams (2018), describió las dificultades de regresar a casa que él y muchos otros veteranos de Vietnam enfrentaron, incluyendo aumentos en la depresión y la ansiedad, a menudo acompañados "con formas debilitantes de adicción" (párr. 2). Las lesiones no físicas como el trastorno de estrés postraumático (TEPT) y las lesiones morales pueden alterar seriamente la identidad y, empañadas por los horrores de la batalla, pueden afectar a los veteranos — si aún no están heridos físicamente de la guerra- mental, emocional y espiritualmente. La expresión creativa es terapéutica para muchos veteranos, lo que les permite resolver las dificultades y el dolor emocional que sentían. "molestar a estos veteranos para hacer estas preguntas básicas sobre estos cambios", dice Adams, "es un elemento importante en el proceso de curación” (párr. 7). Al centrarse en la literatura, o la historia, estos elementos permiten a los veteranos encontrar significado, propósito y comprensión en sus vidas. Adams explica: "Antigua o moderna, la literatura nos da perspectiva, permitiendo que nos veamos más clara y tal vez menos dolorosamente, a través de las experiencias de los demás reales o imaginadas" (párr. 8). Del mismo modo, la historia ayuda a los veteranos a encontrar una conexión entre sus identidades personales en el contexto del tiempo, ayudando tanto a los veteranos como al "país en su conjunto a aceptar su experiencia colectiva" (párr. 11).

Fuera de la guerra, muchos otros también están utilizando el poder curativo de las humanidades. En Europa, Deborah Alma, una poeta galardonada, es conocida como la "Poeta de Emergencia", que ha adoptado un enfoque único para compartir poesía "recetando poemas" para dolencias emocionales a las que conoce (Flood, 2019). Ella cree que la gente está recurriendo más a la poesía en los últimos tiempos para llenar los vacíos dejados por la religión. Sin embargo, independientemente de si las humanidades están basadas espiritual o religiosamente, sin duda fomentan y promueven la curación. El Dr. Patrick Clary es un médico que trabaja con pacientes de hospicio y con frecuencia añade poesía como acompañamiento a sus recetas escritas a mano. Aunque confiesa no ser un practicante de la medicina narrativa - una rama importante 
en las humanidades médicas - admira el inicio de Rita Charon. En su artículo, reflexiona sobre los pensamientos de Charon acerca de la conexión entre la medicina, la curación y el componente espiritual que tiene:

... en la vida moderna, buscamos significado más intensamente cuando estamos amenazados y de alguna manera sufriendo. En otro tiempo, la "pérdida de fe" podría haber provocado un trabajo similar del alma en compañía de los santos. Enfrentando la mortalidad ahora, a menudo nos dirigimos a los médicos en lugar de a los sacerdotes. (Clary, 2010, pp. 797-798)

Aunque los médicos no pueden ni deben asumir necesariamente el papel de un sacerdote, porque la sociedad los mira de la misma manera con respecto a su destino físico, los médicos deben reconocer las dificultades que los pacientes están sufriendo y ser sensibles a sus necesidades. Debido a este gran cambio en la cultura médica y a quien la humanidad recurre para la curación, es importante que la medicina moderna, durante tanto tiempo una esfera centrada científicamente, se abra a las humanidades para complementar los tratamientos y conectar tanto al paciente como al médico entre sí, abrazando y alentando las implicaciones humanitarias en un campo tan complicado. Las humanidades pueden basarse en el avance del conocimiento médico para crear significado, profundidad y conexión entre el médico y el enfermo. Al ayudar a los médicos a involucrarse empáticamente con el sufrimiento del paciente, las humanidades pueden ayudar a sanar a al paciente en su totalidad, en lugar de solo sus síntomas.

Un artículo analiza los efectos de la poesía para los pacientes al final de la vida, explicando que "el objetivo de la atención médica es curar al paciente de su dolor, mientras que la poesía ofrece un medio para sanar el sufrimiento de la persona" (Hovey et al., 2018, p. 197). La poesía no es el único método para lograrlo, ya que las humanidades médicas abarcan también la literatura, el arte, la música y la historia, que también ayudan a los enfermos a desarrollar significado, claridad y perspectiva en sus luchas. Debido a la interconexión del sufrimiento y el dolor, la medicina debe reconocer los muchos aspectos que comprenden el dolor y el sufrimiento para sanar a todo el paciente. Acompañar al enfermo a través de sus ensayos no sólo es posible, sino que se anima a ayudar a los pacientes a sentirse atendidos, y a los médicos a encontrar sentido en sus trabajos. Hacer esto proporcionaría un mejor tratamiento y permitiría a los pacientes sanar física, mental, emocional y espiritualmente.

\section{El paciente}

"En la medicina, como ciencia, la manera adecuada es la objetividad, el rigor y la lógica poco excusadora. La elocuencia, la calidez personal, la simpatía y el temperamento son excentricidades en la ciencia; son la sangre de vida de las humanidades"

(Dolan, 2015, p. 85) 
El beneficiario más obvio de las humanidades médicas es el paciente, pero hay muchas razones para exactamente por qué, ya que hay aspectos psicológicos y sociológicos en él. "Etimológicamente", dice Susan Sontag, "paciente significa enfermo... no sufrir como tal que es más profundamente temido, sino sufrimiento que se degrada” (Sontag, 1977, p. 125). A nivel sociológico, la enfermedad siempre ha causado aislamiento, vergüenza, crisis de identidad, pérdida y miedo (p. 6). En la antigüedad, la enfermedad era un símbolo de pecado y mal carácter, o el resultado de la maldición de un individuo (p. 6). En los tiempos modernos, sin embargo, en lugar de explicar el mal carácter de una persona, la literatura la ha reemplazado por "la noción de que expresa carácter" (p. 46). Sontag escribió algunos ensayos sobre las implicaciones metafóricas de la enfermedad y su impacto en los pacientes. Uno de sus ensayos publicados en los años 70 durante la epidemia de SIDA, donde las implicaciones sociales de la enfermedad llevaron a muchos "a una muerte social que precede a la física" (p. 122). "Las metáforas y mitos", dice al mirar el simbolismo social detrás del SIDA, "estaba convencida, matar" (p. 102). Sontag reflexionó sobre cómo las implicaciones sociales de la adquisición del SIDA trajeron aislamiento, vergüenza y miedo. El juicio social con respecto a los de la comunidad LGBTQ+ en ese momento, dice Sontag, debe haber hecho "la tentación de ocultar un hallazgo positivo... grande" (p. 120). Estas implicaciones sociales a menudo afectan la vida de los pacientes, sus respuestas al tratamiento y, por lo tanto, su capacidad para sanar.

Para muchos pacientes obesos, las implicaciones sociales de un cuerpo sano afectan la forma en que se ven a sí mismos. Un estudio que descubrió las implicaciones sociales en el carácter y la obesidad descubrió que las personas obesas son frecuentemente retratadas en los medios de comunicación con muchas cualidades negativas, lo que hace que muchos de los obesos pierdan su autoestima (Grønning et al., 2013). Otro obstáculo que afecta la capacidad de pacientes como este para sanar es encontrar un médico que no los juzgue y que demuestre un entendimiento por las dificultades que estos pacientes pueden estar enfrentando. Para los pacientes obesos, ir al médico es difícil porque se sienten juzgados por sus proveedores y desanimados porque "la actitud general es que 'sólo tienes que tomar el control”, pero, para muchos, no es tan simple (pp. 276-77). Los pacientes de este estudio expresaron con frecuencia la frustración que sentían con los médicos que no los entendían, a pesar de que los pacientes estaban tratando seriamente de controlar su peso (pp. 275-77). En situaciones como estas y muchas otras, con demasiada frecuencia se escucha que la manera brusca o desconsiderada de un médico junto a la cama o su incapacidad para escuchar o comunicar frustra y aleja a los pacientes.

John Smith, como alguien que ha sido paciente, hizo el siguiente comentario: "Las expectativas del paciente y las expectativas del proveedor médico con mayor frecuencia no se alinean entre sí, y mucho menos comparten el mismo objetivo". Si no se comunican las expectativas del paciente y del proveedor, ninguna de las partes logrará sus objetivos, y el sistema falla. Al fomentar la curación, la empatía y una conexión definitiva entre las partes, 
las humanidades podrían ayudar a facilitar una mejor comunicación entre los médicos y los pacientes. Esto ayudaría a evitar que los médicos hablen con sus pacientes, permitiendo a los pacientes entender sus diagnósticos más plenamente, y establecer confianza con sus proveedores para que puedan hacer más preguntas y abordar sus preocupaciones.

Las humanidades también pueden dar a los pacientes una salida para expresar emociones difíciles que pueden estar experimentando. Por ejemplo, aquellos con afecciones crónicas del dolor pueden encontrar un medio de expresión desenfrenado por cualquier restricción que les permita alcanzar un nivel más profundo de comprensión sobre sí mismos y su condición tal vez mejor de lo que cualquier expresión literal de dolor podría explicar. Hovey et al. (2018), explica cómo la expresión del dolor requiere un lenguaje metafórico, como el de la poesía, para la libertad de expresión y la libertad de sanar:
La invisibilidad del dolor crónico requiere un tipo más profundo de expresión ... Mientras que la narrativa biomédica [como la del cuadro médico de un paciente] trabaja para anestesiar el dolor de la persona y alcanzar una verdad única objetivamente determinada, la narrativa poética sirve como expresión para la afirmación de la persona de sus verdades... [y proporciona] una representación íntima y detallada del dolor de la persona. (p. 197)

Permitir a los pacientes esta oportunidad de expresarse y llegar a un acuerdo con su sufrimiento permite al paciente, en última instancia, encontrar significado, propósito y una manera de sanar. Mangione et al. explica que las humanidades pueden hacer esto proporcionando "un lenguaje indispensable para explorar esa tierra extraña, matizada y a menudo sin sentido llamada la condición humana" (Mangione et al., 2018, p. 633). Proporcionar este lenguaje tanto al paciente como al médico les permite unirse durante y a través del proceso de curación.

\title{
El médico
}

\begin{abstract}
"El Médico completo es aquel que es capaz en las tres dimensiones: es un practicante competente; es compasivo; y es un hombre educado... Un objetivo educativo más realista es abrir la posibilidad de que todos los estudiantes y profesionales vivan en cierta medida en cada uno de estos tres niveles"
\end{abstract}

(Dolan, 2015, p. 152)

Aunque tal vez sea menos reconocido, el médico también necesita que las humanidades sanen, tanto para sanar como para ser sanadas. Los diagnósticos no sólo pueden ser difíciles, sino que muchos obstáculos pueden dificultar que el médico obtenga toda la historia del paciente. Muchos médicos tienen un límite de tiempo sobre cuánto tiempo se supone que deben ver a un paciente para satisfacer las demandas de la clínica. A menudo, también se les desaconseja 
ordenar demasiadas pruebas, o a veces el cirujano, clínica u hospital en el que trabajan no realiza el tipo de cirugía que los pacientes necesitan. Y, por supuesto, el seguro hace que la atención médica sea aún más complicada.

El sistema de salud es un lugar difícil para trabajar, con los muchos obstáculos legales, políticos, sociales y burocráticos que pueden impedir y dictar cómo un médico hace su trabajo. Enfrentar estos intrincados obstáculos a veces puede dificultar que los médicos canalicen ese lado "humano" de la medicina. Además de trabajar en un entorno complejo, el papel del médico también es increíblemente enrevesado. Un médico, además de almacenar y utilizar sus vastos conocimientos médicos, también es a menudo un líder en la comunidad, un filántropo, un guía nutritivo, un entusiasta de la investigación, un individuo bien redondeado y un salvador para los pacientes que sufren. El Doctor W. W. Mayo, padre de los hermanos que fundaron la famosa Clínica Mayo, expresó la idea de que "los médicos asuman el liderazgo social y político en sus comunidades. Ese fue su privilegio y su deber" (Clapesattle, 1941, p. 132). Él creía que un médico debería estar bien redondeado, la expectativa general para los médicos de hoy (Mangione et al., 2018, p. 629). El proceso para entrar en la escuela de medicina es bastante difícil, pero los ensayos no terminan ahí, trabajando para satisfacer las necesidades de muchos pacientes, las necesidades del hospital o la clínica, las responsabilidades de la comunidad, la vida y las responsabilidades personales, y mantenerse al día con los conocimientos médicos que cambian rápidamente, todo puede acumularse y conducir al desánimo y el agotamiento. De hecho, los médicos estadounidenses tienen las tasas de suicidio más altas de cualquier otra profesión en el país, incluso mayores que las de la población general (Kalmoe et al., 2019; Mangione et al., 2018; Turner, 2019). El Dr. Edward Ellison, director médico, declaró en una conferencia sobre cómo la industria de la salud necesita comenzar a implementar programas para ayudar a "enseñar autocuidado y proporcionar apoyo emocional y espiritual” (Turner, 2019, párr. 2). Los médicos tienden a ser personas que, esencialmente, corren hasta que ya no pueden. Esto inevitablemente causa agotamiento. "Uno de los síntomas del agotamiento es la pérdida de propósito, que a menudo se expresa como sensación de que los estudiantes y los practicantes no están haciendo una diferencia en la vida de su paciente" (Childress, 2018, párr. 11).

Para el poeta-médico Rafael Campo, M.D., la poesía le permite recargarse creando un espacio para reconectarse con la humanidad. En una entrevista con la Asociación de Colegios Médicos Americanos, una vez comentó cómo la poesía le permite ser "humano junto" con sus pacientes (Weiner, 2019, párr. 10). Utilizando las humanidades como un medio para recargarse y cuidarse a sí mismos, reavivando la empatía y lo que significa ser humano, los médicos pueden utilizar estas herramientas para reconectarse con sus pacientes experimentando la curación ellos mismos. Por lo tanto, uniendo al paciente y al médico con su humanidad común, ambos pueden alcanzar el nivel deseado de significado, propósito y conexión. 
Sin embargo, a muchos médicos se les enseña desde el principio en la escuela de medicina que cosas como la poesía y la medicina no se mezclan, como el Dr. Campo descubrió en su viaje médico (Weiner, 2019). Otro médico afirmó que mientras estaba en la escuela de medicina, una residente pensó que pasaba demasiado tiempo hablando con su paciente y le preguntó por qué hizo eso. Al describir la experiencia, explicó: "Estaba tratando de ayudarla a sentirse mejor'. Él dijo, 'no es tu trabajo hacerla 'sentir mejor'. ¡No estaba de acuerdo!” Romper esta dicotomía de larga data podría abrir las puertas a un nuevo tipo de cultura médica que es beneficiosa para todos. Al hacerlo, tal vez los médicos y estudiantes de medicina descubrirían conexiones y comprensión entre las disciplinas que nunca pensaron antes. El Dr. Campo explicó la conexión que encontró entre la poesía y la medicina:

Poesía en su corazón es esta experiencia empática compartida no muy diferente del encuentro clínico, donde a menudo nos dedicamos a la experiencia muy privada del sufrimiento de otra persona... La poesía y la medicina son viscerales y físicas. Si cavas un poco más profundo, realmente hay una profunda conexión entre ellos. (Weiner, 2019, párr. 15)

La poesía no es el único medio por el cual se logran estas conexiones. Como las humanidades médicas abarcan modos como la literatura, la historia, el arte y la música, hay muchas maneras en que el médico puede involucrar y encontrar capas de profundidad y significado para la medicina. Al adaptar las humanidades a sus planes de estudio, las escuelas de medicina enseñan a los futuros médicos desde el principio que una educación interdisciplinaria no es tan extraña, y, de hecho, podría ser la clave para llenar el vacío de larga data del sistema de salud.

\section{Humanidades en educación médica}

$\mid$

“El arte y la salud no deben separarse... No están separados, más que la mente y el cuerpo están separados”

(Swedlund, 2019, párr. 7)

Aunque muchas escuelas de medicina requieren clases de ética médica para ayudar a los médicos en formación a discutir circunstancias hipotéticas difíciles donde la relación entre el médico y el paciente es tensa, los escenarios simulados actuales son cortos, sin mucha descripción, y a menudo carecen de la profundidad y ambigüedad de los casos de la vida real (Nash, 2018). También son en su mayoría unilaterales, donde los médicos siempre parecen estar equivocados al ser groseros o descuidados hacia el paciente (p. 191). Un artículo sugiere una manera para que los estudiantes de medicina saquen el máximo partido a estas clases, y para hacer estos casos de ética médica más parecidos a la vida, es que los propios estudiantes de medicina reescriban más contenido en profundidad de las historias y las discutan entre sí (pp. 191, 201, 204). Esto da profundidad y significado a estos escenarios, permitiendo que no haya una 
respuesta correcta — como a menudo no hay en la vida real - y los estudiantes pueden hacer sus propios juicios sobre las situaciones (p. 204). Esta aplicación podría abrir puertas para nuevas perspectivas, perspectivas y conversaciones.

La medicina está lejos de ser una ciencia exacta y, como en la vida real, muchos escenarios a menudo no se resuelven. Hay conflictos de pruebas, síntomas extraños y tratamientos que funcionan para algunos y no para otros. Childress (2018) explicó que, para hacer frente a esto, trabajar en medicina "requiere no sólo tolerancia a la ambigüedad, sino una capacidad para mantener múltiples perspectivas en la mente al mismo tiempo con el fin de discernir qué plan de tratamiento es en el mejor interés del paciente" (párr. 8-9). Al igual que la medicina, la literatura también contiene escenarios de ambigüedad. Por ejemplo, muchas historias describen personajes y escenarios con opiniones y objetivos contradictorios. Se exploran las psicologías de los personajes y las razones por las que toman ciertas decisiones son implícitas o ambiguas, lo que permite a los lectores sacar sus propias conclusiones sobre la situación. A veces no hay una conclusión definitiva para los caracteres o eventos presentados en el texto, y esto permite ambigüedad y también podría ayudar a desarrollar esta capacidad de mantener múltiples perspectivas a la vez. Los proveedores que están familiarizados con el uso de estas técnicas que se encuentran en la literatura podrían ser capaces de mejorar su enfoque de la ambigüedad en los casos médicos.

Un estudio en el que participaron estudiantes de medicina de varias universidades determinó que a medida que los estudiantes estaban más expuestos a las humanidades, eran más propensos a ser sabios, empáticos, abiertos y tolerantes con la ambigüedad (Mangione et al., 2018, p. 630). Todas estas cualidades son importantes, si no esenciales, para los médicos. La encuesta explica cómo el desarrollo de estas cualidades es intuitivo a partir de esta exposición, ya que "las humanidades no sólo son una forma de enseñar compasión y tolerancia, sino que también representan la sabiduría de aquellos que vinieron antes que nosotros” (pp. 631-32). Los estudiantes que estaban más expuestos a las humanidades también eran menos propensos a enfrentar agotamiento manifestándose en fatiga mental, emocional y física (p. 630). En un mundo donde las humanidades son demasiado a menudo "habladas como si fueran una pérdida de tiempo", esta encuesta describe claramente su asociación con muchos rasgos deseados que parecen carecer de la profesión médica actual (p. 632). Como tal, tal vez sea esencial que estas disciplinas juntos para ayudar a los estudiantes, no sólo para alentar estas cualidades personales, sino también para hacer frente al agotamiento que muchos médicos y ciertamente estudiantes de medicina enfrentan.

The University of Arizona College of Medicine (La escuela de medicina de la universidad de Arizona - UACOM) es una escuela que aplica las humanidades médicas en sus planes de estudio. Como muchos otros, la UACOM lo hace colaborando con el programa de humanidades de la universidad. En este caso, la poesía se enseña a los estudiantes de primer año de medicina. La Dra. Ellen Melamed, instructora de la UACOM, explicó en el periódico en línea de la 
universidad cómo el arte y la salud no están más separados que la mente y el cuerpo (Swedlund, 2019). La filosofía de dualismo mente-cuerpo de Descartes probablemente despegó y estableció el estándar para la escuela de medicina de pensamiento que todavía reina hoy en día. Aun así, sería injusto culpar de nuestro abandono pasado de la salud mental a sus filosofías innovadoras (Whitehead et al., 2016). Es desafortunado que haya tomado la medicina el tiempo que tenga que reconocer su conexión, pero estos aspectos no físicos para la salud - mental, emocional y espiritual- finalmente se están enseñando para que los futuros médicos reconozcan su impacto en la salud física. Como explica el Dr. Melamed, el cuerpo en sí se estudia no sólo a través de la medicina, sino también en el arte: "Un escultor tiene que aprender anatomía, y un médico tiene que aprender anatomía. Un bailarín tiene que aprender fisiología, y un médico tiene que aprender fisiología" (Swedlund, 2019, párr. 10). Aunque los método'os son diferentes para lograr estos entendimientos, el hilo conductor entre ellos son los humanos que estos cuerpos albergan. El cuerpo sigue siendo el cuerpo, sólo mirado a través de diferentes alcances. Si estas lentes se combinaran, las capas de significado podrían llevar a los estudiantes de medicina a entender y proporcionar conexiones que tal vez ni siquiera hubieran imaginado previamente. Dada la literalidad de los cursos de ciencias requeridos para la escuela de medicina, estos estudiantes probablemente nunca han explorado el mundo de la metáfora y la alusión. Melamed (2019) comenta que "al traerlos a este espacio, con su sentido de apertura y ligereza, estamos abriendo las mentes de estos médicos en formación para buscar soluciones que tengan en cuenta al paciente en su conjunto" (párr. 5).

Mientras que herramientas como la literatura, la poesía y tal vez la historia permiten al lector tener múltiples perspectivas y ser tolerante con la ambigüedad, cosas como la música, la literatura, la poesía y el arte permiten la creatividad y la imaginación, que son útiles en habilidades como la resolución de problemas. Un estudio realizado entre profesionales de la salud mental y personal de servicios sociales también descubrió que, en un taller de resolución de problemas, las artes permitieron una mejor comunicación profesional y fomentaron la creación de equipos entre los participantes (Acai et al., 2016). $\mathrm{Al}$ tiempo que mejora la comunicación y la creación de equipos, esto podría permitir a los médicos también mejorar sus relaciones y la comunicación entre otros miembros del equipo de atención médica. A medida que los equipos de salud continúan creciendo, abarcando diferentes tipos de terapias, asistentes médicos, enfermeras, auxiliares de enfermería, asistentes médicos, personal quirúrgico, etc., estas habilidades serían críticas en el proceso de educación de los futuros médicos. En la Universidad de Utah ( $U$ de $U$ ), un plan de estudios basado en humanidades se integra en los primeros dos años de clases de su estudiante de medicina. Marcie, estudiante de primer año de medicina, explica cómo el curso se reúne en grupos pequeños cada dos semanas para discutir temas no enseñados en sus estudios científicos o clínicos. En nuestra entrevista, hizo el siguiente comentario: 


\begin{abstract}
Escuchar y compartir diferentes puntos de vista con mis compañeros es valioso para que pueda tener una visión más amplia de estos temas. Esto me ayuda a prepararme para abordar temas difíciles con los pacientes. También me siento más consciente de las injusticias en nuestro sistema de salud y me siento más preparado para abogar por poblaciones marginadas o desatendidas.
\end{abstract}

Ella dice que la escuela de medicina también fomenta un ambiente donde los estudiantes saben que pueden pedir ayuda si la necesitan. El hospital U de U también tiene muchos programas donde los pacientes pueden tomar clases de música o arte. Marcie dice: "Esto ayuda a los pacientes a sobrellevar y encontrar alegría cuando otras partes de sus vidas son tomadas debido a su enfermedad". Más programas y un plan de estudios infundido por humanidades, como los de la UACOM y la $U$ de $U$, permiten a los estudiantes de medicina y pacientes a nivel clínico entender cómo las humanidades pueden ayudar tanto a los pacientes como a los proveedores a lograr la curación.

\title{
Atención holística
}

"La filosofía de la medicina osteopática se centra en la conexión del cuerpo humano y de la sociedad. La atención holística es el resultado de esta filosofía, un tipo de atención que se refiere no sólo a los aspectos físicos de la enfermedad, sino también a sus características emocionales, mentales, sociales y espirituales"

(profesor de la Universidad Rocky Vista)

El propósito de integrar las humanidades en la educación médica es lograr una atención holística en la clínica. Un profesor del Rocky Vista University College of Osteopathic Medicine (o RVU) describió cómo la atención holística se centra en el tratamiento no sólo de los síntomas físicos que indican enfermedades, sino en cómo las facetas emocionales, mentales y espirituales afectan la salud general. "Además", añade este profesor, "la atención holística requiere un alto nivel de pensamiento crítico, ya que hacerlo requiere que los profesionales de la salud equilibren muchas ideas, deseos y valores que compiten”. Las humanidades médicas fomentan este enfoque de atención holística, y se esfuerza por lograrlo mediante su integración de las humanidades en las escuelas de medicina. "Las humanidades médicas", explica este profesor de RVU, "buscan dotar a los profesionales de la salud de un mejor pensamiento crítico e imaginación moral, habilidades que serán absolutamente necesarias en la práctica médica futura”. A medida que la cultura médica está cambiando, estas habilidades que fomentan las humanidades se convertirán en fundamentales para ayudar a los médicos en sus futuras prácticas. La discusión hasta ahora se ha centrado en esta integración en las escuelas tradicionales, o alopáticas, de medicina. Sin embargo, hay otro camino menos conocido que un médico en formación estadounidense puede tomar donde su filosofía enfatiza la atención holística, esta es la medicina osteopática. 
La filosofía de un Doctor en Medicina Osteopática (o D.O.) incorpora estos aspectos no físicos de la salud en la formación del médico. Lo hace haciendo hincapié en el bienestar y la medicina preventiva, no sólo en el tratamiento de los síntomas de un paciente. El sitio web de RVU explica cómo los D.O. se centran en trabajar con los pacientes para establecer estilos de vida saludables para prevenir enfermedades, un área muy necesaria en la atención médica (Rocky Vista University, 2020, párr. 18). Los D.O. también tienen un entrenamiento adicional único en lo que se llama medicina manipuladora osteopática, donde "los médicos usan sus manos para diagnosticar enfermedades y lesiones y para fomentar la tendencia natural de su cuerpo hacia una buena salud" (párr. 8). Al observar los aspectos físicos y no físicos de la salud, el paciente entero puede curarse. "Cuando miramos las historias de los pacientes, estamos tratando a toda la persona", explicó el Dr. Melamed, "no estamos tratando el riñón en la habitación 206” (Swedlund, 2019, párr. 9). Los estudiantes de medicina de RVU, como resultado de esta atención holística, están obligados a tomar cursos que se centren en las humanidades médicas. "Estos cursos", explica otro profesor de RVU, "comprenden el programa de Ética Médica, el programa de Educación Interprofesional y el programa de Medicina Basada en evidencia. Además, hay varias clases electivas basadas en las Humanidades Médicas como Historia de la Medicina y la Literatura y la Medicina”. Las humanidades amplían la capacitación médica formal de los médicos de tal manera que les permite entender y tratar a todo el paciente, mirando todas las maneras en que los pacientes pueden y necesitan sanar. Escuelas como la de la medicina osteopática, se esfuerzan por integrar esto en su educación. Incorporar aún más estos ideales de atención holística en las escuelas de medicina alopáticas impulsaría este enfoque en la formación de los estudiantes de medicina para, en última instancia, llenar las lagunas en el sistema de salud.

RVU recientemente abrió un segundo campus en el sur de Utah, donde los médicos han estado estudiando medicina bajo las montañas de roca roja de Ivins. La universidad proporciona una Maestría en Ciencias Biomédicas, así como un Doctorado en Medicina Osteopática, que ambos enfatizan un enfoque de atención holística. Al enfatizar esto en su educación, los futuros médicos que vienen al sur de Utah están participando en aspectos clave que las humanidades se esfuerzan por enseñar. La incorporación de las humanidades médicas en este enfoque holístico les permite participar más en la comunidad. Este profesor menciona además cómo uno de los valores fundamentales de RVU es la compasión, razón por la cual la escuela alienta a los estudiantes a participar en la comunidad en lugares como Switchpoint, un centro para personas sin hogar en St. George, así como impulsos para artículos necesarios, esfuerzos de limpieza y ayudar a los niños desfavorecidos en edad escolar. Este enfoque centrado en la holística fomenta esta compasión, y a medida que los estudiantes se involucran en la comunidad, están participando en este valor básico que los ayudará en sus futuras carreras como médicos. 
En todo el país, hay una notable escasez de medicamentos preventivos y proveedores de atención primaria que sólo seguirán creciendo a medida que las generaciones mayores continúen envejeciendo (Health Care Trends, 2018). Mientras que la mayoría de los médicos centrados en los alopáticos suelen estar más preocupados por la investigación y la especialización, los D.O. se centran en la atención primaria, familiar y geriátrica, aunque un D.O. puede especializarse en cualquier cosa. Para St. George Utah, el enfoque en la familia, la geriatría y el cuidado del final de la vida, como señala un profesor de RVU, son "áreas en las que el condado de Washington tiene necesidades particulares". El enfoque holístico de atención que toman las escuelas osteopáticas no sólo llena esta brecha nacional entre proveedores y pacientes, sino también esa brecha evidente entre la humanidad de la medicina y la atmósfera actual del sistema de salud. A medida que esto comienza a incorporarse al condado de Washington, estas áreas tan necesarias podrían ser cubiertas y llevar atención competente a los ciudadanos y proporcionar significado y satisfacción para los médicos que incorporan esta filosofía a su cuidado.

\section{Innovaciones y aplicaciones al condado de Washington}

"Por "sanación" me refiero simplemente a cruzar el umbral tradicional de un complejo médico-industrial y comenzar a interactuar con el paciente, con su historia, en su territorio, en su casa, y comprometerse con sus familias y seres queridos y sus historias"

(Dolan, 2015, p. 231)

Si el condado de Washington continúa implementando las filosofías de una atención holística, tanto sus ciudadanos como sus pacientes se beneficiarán enormemente. Muchos educadores del condado de Washington y afiliados del Dixie Regional Medical Center fueron entrevistados para esta investigación, y todos expresaron cómo la incorporación de las humanidades médicas literatura, poesía, música, arte e historia - podría beneficiar significativamente a la comunidad. Uno mencionó cómo "veríamos un gran cambio en la salud de nuestra comunidad", mientras que otro mencionó que "las Humanidades Médicas proporcionan una construcción y un marco en el que [la curación] se puede lograr”. Además de proporcionar un medio para ayudar a los pacientes a sanar en un entorno de atención médica, y para que los médicos cumplan con su potencial como cuidadores, muchos participantes de este estudio también expresaron cómo cosas como la lectura, la meditación, la música, el arte y la historia les ayudan a hacer frente a los desafíos, entender sus situaciones actuales e involucrarlos con las personas que los rodean.

John Smith también hizo la interesante observación de que los ciudadanos estadounidenses, en comparación con otros países, no están tan médicamente alfabetizados. Esto significa que los pacientes no están preparados para sus citas médicas, lo que hace que sea más difícil para ellos entender y hacer preguntas sobre sus circunstancias. Otro participante mencionó cómo los pacientes 
sienten presión para permanecer en silencio en sus citas, aceptando - en la clínica, de todos modos - la voluntad del médico, sin acatar el consejo médico porque no están convencidos de que funcione. Si los sistemas educativos en el condado de Washington incorporaran una manera de enseñar a los estudiantes o a sus miembros de la comunidad cómo navegar por el lenguaje médico, tal vez se sentirían más cómodos hablando y comunicarse con sus proveedores. Del mismo modo, si los médicos se dedican a recanalizar ese lado humano de la medicina, posiblemente incorporando las humanidades médicas en las clínicas, pueden participar empáticamente en el sufrimiento de sus pacientes y conectarse mejor con ellos. Del mismo modo, si los médicos se dedican a recanalizar ese lado humano de la medicina, como mediante la incorporación de las humanidades médicas en la educación y las clínicas, tal vez sean más conscientes de las condiciones de sus pacientes y no hablen con ellos porque se han dedicado empáticamente al sufrimiento de sus pacientes.

Médicos como el anteriormente mencionado Dr. Campo, el Dr. Clary, junto con muchos otros, han utilizado cosas como la poesía en sus entornos clínicos. Mientras que el Dr. Clary ha proporcionado poesía y lecciones de vida a sus pacientes de hospicio, el Dr. Campo organiza un taller de poesía en su clínica para ayudar a los pacientes a contar las historias de sus enfermedades (Clary, 2010; Weiner, 2019). Si bien no todos los médicos pueden hacer lo que estos médicos están haciendo en un sentido tan literal, hay terapias alternativas que pueden incorporar estos elementos en el tratamiento. Acompañar el tratamiento médico con cosas como el arte, los ejercicios de escritura reflexiva, escuchar música, recitar poesía o buscar momentos históricos que se correlacionen con la difícil situación del paciente, puede involucrar a los pacientes en humanidades médicas. Smith afirma que su terapia ocupacional y física ha incorporado cosas como música y tocar el piano para ayudar en el proceso de curación. Otro participante mencionó cómo la historia le permite dar sentido a la actual pandemia de COVID comparándola con la epidemia de gripe española de 1918, que también utilizó medidas de distanciamiento social utilizadas hoy en día.

Otro participante mencionó, "si usted puede conducir a casa ese mensaje [del poder de la conexión humana] entonces usted puede arreglar el problema.” Esto se puede hacer a través de las humanidades médicas: exposición e incorporación de literatura, poesía, música, arte e historia. La integración de las humanidades médicas en la educación y los entornos clínicos del condado de Washington puede beneficiar tanto al paciente como al médico, lo que les permite unirse en su humanidad común para promover el significado, el propósito y la curación. 


\section{References}

Acai, A., Mcqueen, S. A., Fahim, C., Wagner, N., McKinnon, V., Boston, J., Maxwell, C., \& Sonnadara, R. R. (2016). 'It's not the form; it's the process': A phenomenological study on the use of creative professional development workshops to improve teamwork and communication skills. Medical Humanities, 42(3), 173-180. https://doi.org/10.1136/medhum-2015-010862

Adams, W. (2018, May). Healing the wounds of war. The Andrew W. Mellon Foundation Blog. https://mellon.org/shared-experiences-blog/humanities-and-experience-war/\#top

Aesculapius. (1922). The British Medical Journal, 2(3227), 882-883.

Childress, A. (2018). Medical humanities and the search for meaning. Baylor College of Medicine. https://blogs.bcm.edu/2018/07/11/medical-humanities-and-the-search-for-meaning/

Clapesattle, H. (1941). The doctor's mayo. Oxford University Press.

Clary, P. L. (2010). Poetry and healing at the end of life. Journal of Pain and Symptom Management, 40(5), 796-800. https://doi.org/10.1016/j.jpainsymman.2010.05.005

Dolan, B. (Ed.). (2015). Humanitas: Readings in the development of the medical humanities. University of California Medical Humanities Press.

Flood, A. (2019, January 25). Poetry pharmacy set to open in Shropshire. https://www.theguardian.com/books/2019/jan/25/poetry-pharmacy-set-to-open-in-shropshire

Grønning, I., Scambler, G., \& Tjora, A. (2013). From fatness to badness: The modern morality of obesity. Health: An Interdisciplinary Journal for the Social Study of Health, Illness and Medicine, 17(3), 266-283. https://doi.org/10.1177/1363459312447254

Health Care Trends. (2018, February 19). Is there a shortage of primary care physicians? Evaluating the claims. St. George University School of Medicine. https://www.sgu.edu/blog/medical/is-there-ashortage-of-primary-care-physicians/

Hovey, R. B., Khayat, V. C., \& Feig, E. (2018). Cathartic poetry: Healing through narrative. The Permanente Journal. https://doi.org/10.7812/tpp/17-196

Huang, S.-T., Good, M., \& Zauszniewski, J. A. (2010). The effectiveness of music in relieving pain in cancer patients: A randomized controlled trial. International Journal of Nursing Studies, 47(11), 1354-1362. https://doi.org/10.1016/j.ijnurstu.2010.03.008

Kalmoe, M. C., Chapman, M. B., Gold, J. A., \& Giedinghagen, A. M. (2019). Physician suicide: A call to action. Journal of the Missouri State Medical Association, 116(3), 211-216.

Linggonegoro, D. W. (2018, September 27). How doctors use poetry. Nautilus. https://nautil.us/ issue/64/the-unseen/how-doctors-use-poetry?platform=hootsuite

Mangione, S., Chakraborti, C., Staltari, G., Harrison, R., Tunkel, A. R., Liou, K. T., Cerceo, E., Voeller, M., Bedwell, W. L., Fletcher, K., \& Kahn, M. J. (2018). Medical students' exposure to the humanities correlates with positive personal qualities and reduced burnout: A multi-institutional U.S. survey.Journal of General Internal Medicine, 33(5), 628-634. https://doi.org/10.1007/ $\underline{\text { s11606-017-4275-8 }}$

Muir, S. (2018, August 21). NEH impact: Engaging veterans in alternative therapy. National Humanities Alliance Blog. https://www.nhalliance.org/ neh_impact_engaging_veterans_in_alternative_therapy

Nash, W. (2018). Showing that Medical Ethics Cases Can Miss the Point: Rewriting Short Stories as Cases. Literature and Medicine, 36(1), 190-207. https://doi.org/10.1353/lm.2018.0008

Robinson, V. (1931). The story of medicine. Tudor Publishing Company.

Rocky Vista University. (2020). RVU. https://www.rvu.edu

Sontag, S. (1977). Illness as metaphor and AIDS and its metaphors. Picador. 
Swedlund, E. (2019, May 15). Future doctors open their minds to poetry. UANews.

https://uanews.arizona.edu/story/future-doctors-open-their-minds-

poetry?fbclid=IwAR 2cryyUf $49 \mathrm{zPWmcKa} 12 \mathrm{tHsXYm}-$

aXJHgEdWmolMEtaFrTZs5QugRD72D68

The Caduceus of Mercury and the Staff of Aesculapius. (1924). The British Medical Journal, 1(3305), 794.

Turner, A. (2019). Stress and rigorous work schedules push a doctor to commit suicide every day in the US: "We need them, but they need us." CNBC Events Newsletter. https://www.cnbc.com/ 2019/05/21/stress-and-rigorous-work-push-a-doctor-to-commit-suicide-every-day-in-us.html

Wassiliwizky, E., Koelsch, S., Wagner, V., Jacobsen, T., \& Menninghaus, W. (2017). The emotional power of poetry: Neural circuitry, psychophysiology, and compositional principles. Social Cognitive and Affective Neuroscience, 12(8), 1229-1240. https://doi.org/10.1093/scan/nsx069

Weiner, S. (2019, August 16). The healing power of poetry. AAMC. https://www.aamc.org/newsinsights/healing-power-poetry

Whitehead, A., Woods, A., Atkinson, S., Macnaughton, J., \& Richards, J. (Eds.). (2016). The Edinburgh companion to the critical medical humanities. Edinburgh University Press. 